\title{
CORRELAÇÕES FENOTÍPICAS E GENÉTICO-ADITIVAS EM MARACUJÁ-AMARELO PELO DELINEAMENTO I
}

\section{Phenotypic and genetic-additive correlations in yellow passion fruit obtained by design I}

\author{
Gustavo Menezes Gonçalves ${ }^{1}$, Alexandre Pio Viana ${ }^{2}$, Luciléa Silva dos Reis ${ }^{1}$, \\ Francisco Valdevino Bezerra Neto ${ }^{3}$, Antônio Teixeira do Amaral Júnior ${ }^{2}$, Laudirléa Silva dos Reis ${ }^{4}$
}

\begin{abstract}
RESUMO
Objetivou-se, neste estudo, avaliar o grau de associação entre características de importância econômica de uma população de maracujá-amarelo (Passiflora edulis Sims f. flavicarpa Deg.) por meio da estimação dos coeficientes de correlação fenotípica e genético-aditivas. Cento e treze progênies de maracujá-amarelo foram obtidas conforme Delineamento I e avaliadas nas localidades de Viçosa, MG, e Miracema, RJ, em arranjos em três agrupamentos, com delineamentos em blocos ao acaso, com três repetições e três plantas por parcela. A característica número de frutos por planta demonstrou estar associada negativamente com peso, comprimento e largura de frutos, e positivamente com espessura de casca. Além disso, a característica peso de frutos apresentou correlações fenotípicas e genético-aditivas positivas com todas as demais, excluindo-se número de frutos por planta. Assim, o aumento nas dimensões do fruto pode resultar em diminuição da produção em números de frutos, de modo que métodos mais elaborados de seleção no melhoramento podem ser necessários para que se atinja uma população com alta produtividade e boas características comerciais. Por conseguinte, o uso de índices de seleção pode contribuir para obtenção de ganhos simultâneos em características de importância econômica em maracujá-amarelo.
\end{abstract}

Termos para indexação: Passiflora, melhoramento, seleção genotípica.

\section{ABSTRACT}

The aim of this research was to evaluate the association among economically important traits of a yellow passion fruit population (Passiflora edulis Sims f. flavicarpa Deg.) through the estimate of the phenotypic and genetic-additive correlations. Hundred thirteen yellow passion fruit progenies were obtained by using nested design and evaluated in two environments, Viçosa, MG, and Miracema, RJ, in Brazil, grouped in three sets in a randomized block design, with three replications and three plants per plot. The trait number of fruits per plant demonstrated to be associated negatively with weight, length and width of fruits, and positively with peel thickness. Moreover, the trait weight of fruits expressed positive values for phenotypic and genetic-additive correlations with others, excluded number of fruits by plant. Thus, the increase in the dimensions of fruit may result in decrease of the production in numbers of fruits, so that elaborated methods of selection may be necessary for a high productivity population and good commercial traits. Consequently, the use of selection indexes can contribute to obtain genetic gain in traits of economical importance in yellow passion fruit.

Index terms: Passiflora, breeding, genotypic selection.

(Recebido em 14 de junho de 2007 e aprovado em 25 de março de 2008)

\section{INTRODUÇÃO}

O Brasil ocupa a posição de maior produtor e consumidor mundial de maracujá, sendo produzidos atualmente no país cerca de $450 \mathrm{mil}$ toneladas, o que representa aproximadamente $56 \%$ da produção mundial dessa fruta (ITI TROPICALS, 2007).

Os programas de melhoramento surgem como ferramentas básicas para a busca de genótipos mais produtivos e, assim, proporcionar maior competitividade ao mercado nacional.

O conhecimento da associação entre as características é importante para a consecução de um programa de melhoramento bem planejado. As correlações são levadas em consideração na escolha dos métodos de melhoramento quando se formulam estratégias de seleção simultânea para as várias características estudadas,

\footnotetext{
'Engenheiros Agrônomos, Doutorandos em Genética e Melhoramento de Plantas - Centro de Ciências e Tecnologias Agropecuárias/CCTA - Laboratório de Melhoramento Genético Vegetal/LMGV - Universidade Estadual do Norte Fluminense Darcy Ribeiro/UENF - Avenida Alberto Lamego, 2000 - Parque Califórnia - 28013-602 - Campos dos Goytacazes, RJ - gustavog@uenf.br; lucileasreis@yahoo.com.br

${ }^{2}$ Engenheiros Agrônomos, D.Sc.I, Professores - Centro de Ciências e Tecnologias Agropecuárias/CCTA - Laboratório de Melhoramento Genético Vegetal/LMGV - Universidade Estadual do Norte Fluminense Darcy Ribeiro/UENF - Avenida Alberto Lamego, 2000 - Parque Califórnia - 28013-602 Campos dos Goytacazes, RJ - pirapora@uenf.br, amaraljr@uenf.br

${ }^{3}$ Doutorando em Genética e Melhoramento de Plantas - Centro de Ciências e Tecnologias Agropecuárias/CCTA - Laboratório de Melhoramento Genético Vegetal/LMGV - Universidade Estadual do Norte Fluminense Darcy Ribeiro/UENF - Avenida Alberto Lamego, 2000 - Parque Califórnia - 28013-602 Campos dos Goytacazes, RJ - fvbn@uenf.br

${ }^{4}$ Graduanda em Ciências do Sociais - Centro de Ciências do Homem/CCH - Laboratório de Estudo do Espaço Antrópico/LEEA - Universidade Estadual do Norte Fluminense Darcy Ribeiro/UENF - Avenida Alberto Lamego, 2000 - Parque Califórnia - 28013-602 - Campos dos Goytacazes, RJ -
} leareis@yahoo.com.br 
predizendo a alteração na média de uma característica quando se seleciona em outra.

No estudo da associação entre características, o mais apropriado é o uso dos coeficientes de correlação, pois eles são adimensionais, o que permite a comparação entre diferentes pares de características, diferentemente das covariâncias. As correlações fenotípicas encontradas para duas características quaisquer possuem causas genéticas e ambientais, porém somente as associações de natureza genética são herdáveis.

De acordo com Falconer (1987), a variância genética aditiva é a principal causa da semelhança entre parentes, pois é a parte da variação genética herdável, logo, ela é o determinante principal das propriedades genéticas de uma população e da resposta da população à seleção.

Utilizando-se o Delineamento I de Comstock \& Robinson (1948), podem-se estimar os coeficientes de correlação genética aditiva (FURTADO, 1996), ou seja, a fração herdável da correlação genética. Poucos são os trabalhos com o Delineamento I, entre eles podem-se citar Furtado (1996), Gonçalves et al. (2007) e Pereira \& Amaral Júnior (2001), respectivamente com as culturas do milho, maracujá-amarelo e milho pipoca.

Objetivou-se, neste estudo, avaliar o grau de associação entre as características de uma população de maracujá-amarelo (Passiflora edulis Sims f.flavicarpa Deg.) por meio da estimação dos coeficientes de correlação fenotípica e genético-aditivas.

\section{MATERIALE MÉTODOS}

Foram instalados experimentos em duas localidades: Viçosa-MG (2045'S e 42 $53 \mathrm{~W}$, altitude de $648 \mathrm{~m})$ e Miracema-RJ ( $21^{\circ} 25^{\prime} \mathrm{S}$ e $42^{\circ} 12^{\prime} \mathrm{W}$, altitude de $\left.137 \mathrm{~m}\right)$, em novembro de 2002, com data final de avaliação em maio de 2004. Nesses experimentos, avaliaram-se 113 progênies de maracujá-amarelo provenientes de cruzamentos que seguiram o Delineamento I, procedimento proposto por Comstock \& Robinson (1948), entre plantas de área de plantio comercial em Campos dos Goytacazes-RJ, no período de maio a julho de 2002. Essa população foi composta pelas seguintes variedades: Maguary, Yellow Master e seleções de São Francisco do Itabapoana, com coeficiente de endogamia $\mathrm{F}=0$. Uma planta doadora de grãos de pólen foi cruzada com um grupo de cinco plantas receptoras, formando progênies de irmãos completos e meios-irmãos, conforme Delineamento I. O delineamento estatístico utilizado foi em blocos ao acaso, em que cada parcela foi formada por três plantas, com as progênies (tratamentos) arranjadas em três agrupamentos: 1) progênies de 01 a 40;2) progênies de 41 a 80 e 3) progênies de 81 a 113 . Os agrupamentos constituíram grupos de tratamentos, sendo cada grupo composto por três repetições.

As avaliações das progênies foram efetuadas durante todo o ciclo de produção da cultura, por meio das seguintes características: número de frutos por planta (NF) - avaliado pela contagem do número de frutos provenientes da primeira florada, dividido pelo número de plantas da parcela; peso de frutos (PF) - valor médio obtido, em gramas, pela pesagem de amostras de 25 frutos por parcela; comprimento de frutos $(\mathrm{CF})$ - valor médio obtido, em mm, pela medição da dimensão longitudinal de amostras de 25 frutos por parcela; largura de frutos (LF) - valor médio obtido, em mm, pela medição da dimensão transversal de amostras de 25 frutos por parcela; espessura de casca (EC) - valor médio obtido, em mm, pela medição da dimensão da casca na porção mediana dos frutos (cortados transversalmente) de amostras de 25 frutos por parcela; número de dias até a antese (DA) - estabelecida por meio da contagem do número de dias transcorridos desde o plantio em campo, até a abertura da primeira flor na parcela.

Para as análises de variância de cada uma das características avaliadas, utilizaram-se: nas análises individuais dos ambientes, o modelo genético-estatístico empregado por Cruz \& Carneiro (2003), Furtado (1996) e Gonçalves (2005); na análise conjunta dos ambientes o modelo utilizado por Gonçalves (2005), Hallauer \& Miranda Filho (1988) e Pereira \& Amaral Júnior (2001).

Estimaram-se os coeficientes de correlação fenotípica $\left(r_{F}\right)$ conforme descrito por Cruz et al. (2004):

$$
r_{F}=\frac{\mathrm{PMP}_{x y}}{\sqrt{\mathrm{QMP}_{\mathrm{x}} \cdot \mathrm{QMP}_{\mathrm{y}}}}
$$

em que:

$\mathrm{PMP}_{\mathrm{xy}}=$ produto médio entre as progênies em relação às características $\mathrm{x}$ e $\mathrm{y}$;

$\mathrm{QMP}_{\mathrm{x}}$ e $\mathrm{QMP}_{\mathrm{y}}=$ quadrado médio das progênies em relação às características $\mathrm{x}$ e $\mathrm{y}$, respectivamente.

Os coeficientes de correlação genético-aditivas $\left(r_{\mathrm{GA}}\right)$ entre duas características, $\mathrm{x}$ e y, foram estimados de acordo com o procedimento relatado por Furtado (1996) e Gonçalves (2005), ou seja:

$$
\mathrm{r}_{\mathrm{GA}}=\frac{\hat{\sigma}_{\mathrm{A}_{\mathrm{xy}}}}{\sqrt{\hat{\sigma}_{\mathrm{A}_{\mathrm{x}}}^{2} \cdot \hat{\sigma}_{\mathrm{A}_{\mathrm{y}}}^{2}}}
$$

em que:

$\hat{\sigma}_{\mathrm{A}_{\mathrm{Xy}}}=$ estimador da covariância genético-aditiva entre duas caraçterísticas;

$\hat{\sigma}_{\mathrm{A}_{\mathrm{X}}}^{2}$ e $\hat{\sigma}_{\mathrm{A}_{\mathrm{y}}}^{2}=$ estimadores da variância genético-aditivas em relação às características $\mathrm{x} e \mathrm{y}$, respectivamente. 
Os estimadores da variância genético-aditivas foram descritos em Cruz \& Carneiro (2003) e Falconer (1987) sendo $\hat{\sigma}_{\mathrm{A}}^{2}=4 \hat{\sigma}_{\mathrm{M}}^{2}$, quando se considera ausência de epistasia e uma população não endogâmica $(\mathrm{F}=0)$. A partir dos valores dos quadrados médios, obtidos pela análise de variância dos dados de Viçosa e Miracema, estimou-se o componente de variância associado ao efeito de macho $\left(\hat{\sigma}_{M}^{2}\right)$, conforme descrito em Cruz \& Carneiro (2003), Falconer (1987) e Gonçalves (2005).

De acordo com Furtado (1996), a covariância genético-aditiva entre dois indivíduos pode ser estimada de maneira semelhante ao componente de variância genético-aditiva, sendo fornecida pelo seguinte estimador $\hat{\sigma}_{\mathrm{A}_{\mathrm{xy}}}=4 \hat{\sigma}_{\mathrm{M}_{\mathrm{xy}}}$, onde $\hat{\sigma}_{\mathrm{M}_{\mathrm{xy}}}$ é a covariância em nível de machos.

Avaliou-se a hipótese de que o coeficiente de correlação (r) ser igual a zero pela estatística “ $t$ ”, segundo Cruz et al. (2004), pela expressão:

$$
\mathrm{t}=\frac{\mathrm{r}}{\sqrt{1-\mathrm{r}^{2}}} \sqrt{\mathrm{n}-2}
$$

Em que "t" está associado a n-2 graus de liberdade e ao nível de $1 \%$ de probabilidade, sendo n igual ao número de pares de observações.

\section{RESULTADOS E DISCUSSÃO}

A Tabela 1 apresenta as estimativas dos coeficientes de correlação fenotípica e genético-aditivas, para o ambiente de Viçosa. Verifica-se que a característica número de frutos por planta apresentou correlação fenotípica e genético-aditiva negativa significativa com as características peso, comprimento e largura de frutos, indicando que a seleção para aumentar o número de frutos por planta pode levar a uma redução no seu peso e tamanho, contradizendo os valores encontrados por Viana et al. (2003), que estimaram correlação genética positiva e significativa entre número e peso de frutos para o ambiente de Campos dos Goytacazes $(0,59)$. Porém, há diferenças nos tipos de correlações calculadas nesses trabalhos, o primeiro com correlação genético-aditiva e o outro com correlação genética. Contudo, deve-se ressaltar que as correlações genotípicas são próprias de uma população em estudo e, por conseguinte, não é recomendada sua extrapolação.

Pode ser evidenciado também que um aumento no número de frutos por planta (NF) resultaria em aumento na porcentagem de polpa, verificado pela correlação positiva significativa $(0,18)$ entre essas duas características, e frutos com maior NF apresentaram um menor número de dias até

Tabela 1 - Estimativas dos coeficientes de correlação fenotípica $\left(r_{F}\right)$ e dos coeficientes de correlação genético-aditivas $\left(\mathrm{r}_{\mathrm{GA}}\right)$ entre sete características de maracujá-amarelo para o ambiente de Viçosa.

\begin{tabular}{|c|c|c|c|c|c|c|c|}
\hline Características $^{1 /}$ & & PF & $\mathrm{CF}$ & LF & $\mathrm{EC}$ & DA & PP \\
\hline \multirow[t]{2}{*}{$\mathrm{NF}$} & $\mathrm{r}_{\mathrm{F}}$ & $-0,31 * *$ & $-0,27 * *$ & $-0,22 * *$ & $-0,02^{\mathrm{ns}}$ & $-0,57 * *$ & $0,10^{\mathrm{ns}}$ \\
\hline & $\mathrm{r}_{\mathrm{GA}}$ & $-0,87 * *$ & $-0,89 * *$ & $-0,82 * *$ & $-0,11^{\mathrm{ns}}$ & $-0,11^{\mathrm{ns}}$ & $0,18 * *$ \\
\hline \multirow[t]{2}{*}{$\mathrm{PF}$} & $\mathrm{r}_{\mathrm{F}}$ & - & $0,76^{* *}$ & $0,83 * *$ & $0,27 * *$ & $0,24 * *$ & $-0,13^{\mathrm{ns}}$ \\
\hline & $\mathrm{r}_{\mathrm{GA}}$ & - & $0,94 * *$ & $0,86^{* *}$ & $0,62 * *$ & $0,51 * *$ & $-0,27 * *$ \\
\hline \multirow[t]{2}{*}{$\mathrm{CF}$} & $\mathrm{r}_{\mathrm{F}}$ & & - & $0,64 * *$ & $-0,08^{\mathrm{ns}}$ & $0,41 * *$ & $-0,04^{\mathrm{ns}}$ \\
\hline & $\mathrm{r}_{\mathrm{GA}}$ & & - & $0,82 * *$ & $0,35^{* *}$ & $0,66 * *$ & $0,06^{\mathrm{ns}}$ \\
\hline \multirow[t]{2}{*}{$\mathrm{LF}$} & $\mathrm{r}_{\mathrm{F}}$ & & & - & $0,22 * *$ & $0,28 * *$ & $-0,21 * *$ \\
\hline & $\mathrm{r}_{\mathrm{GA}}$ & & & - & $0,45^{* *}$ & $0,70 * *$ & $-0,35 * *$ \\
\hline \multirow[t]{2}{*}{$\mathrm{EC}$} & $\mathrm{r}_{\mathrm{F}}$ & & & & - & $-0,23 * *$ & $-0,46^{* *}$ \\
\hline & $\mathrm{r}_{\mathrm{GA}}$ & & & & - & $-0,10^{\mathrm{ns}}$ & $-0,08^{\mathrm{ns}}$ \\
\hline \multirow[t]{2}{*}{ DA } & $\mathrm{r}_{\mathrm{F}}$ & & & & & - & $0,07^{\mathrm{ns}}$ \\
\hline & $\mathrm{r}_{\mathrm{GA}}$ & & & & & - & $-0,27 * *$ \\
\hline
\end{tabular}

${ }^{1 / N F}$ : número de frutos por planta; PF: peso de frutos, em gramas; CF: comprimento de frutos, em mm; LF: largura de frutos, em mm; EC: espessura de casca, em mm; DA: número de dias até a antese; PP: porcentagem de polpa.

** Significativo a $1 \%$ de probabilidade pelo teste $\mathrm{t}$.

${ }^{\mathrm{ns}}$ Não significativo. 
a antese, verificado pela correlação fenotípica negativa $(-0,57)$ entre essas características.

A característica peso de frutos apresentou correlações fenotípicas e genético-aditiva positiva significativas para com comprimento de frutos, largura de frutos, espessura de casca e número de dias até a antese e correlações negativas com número de frutos por planta e porcentagem de polpa, denotando que um aumento no peso de frutos possivelmente resultaria em ganhos no comprimento, na largura, na espessura de casca dos frutos e no número de dias até a antese, além de redução no número de frutos por planta e na porcentagem de polpa. Viana et al. (2003) também encontraram correlação genética positiva entre peso de frutos e comprimento de frutos, largura de frutos e espessura da casca, avaliando 20 genótipos de maracujá-amarelo, nos ambientes de Macaé e Campos dos Goytacazes.

As correlações genético-aditivas também ratificaram que um aumento no comprimento de frutos resultaria em igual ação na largura de frutos, espessura de casca e no número de dias até a antese. E aumento na largura de frutos também afeta positivamente as características espessura de casca e número de dias até a antese, e minimiza porcentagem de polpa, dificultando o trabalho de melhoramento, já que o aumento em tamanho dos frutos (comprimento e largura) resulta também em aumento na espessura de casca e no número de dias até a antese, características essas que se deseja diminuir valores, e ainda reduzir em porcentagem de polpa.

As estimativas das correlações fenotípicas e genéticoaditivas para o ambiente de Miracema estão apresentadas na Tabela 2. Conforme verificado para o ambiente de Viçosa, em Miracema a característica número de frutos por planta demonstrou correlação fenotípica e genético-aditiva negativa com peso e comprimento de frutos, porém de menor magnitude, e não significativa com espessura de casca e número de dias até a antese. Divergentemente do ambiente de Viçosa, essa característica mostrou correlação genéticoaditiva positiva com largura de frutos.

A característica peso de frutos apresentou-se correlacionada positivamente com comprimento de frutos, largura de frutos e número de dias até a antese. Entretanto, diferentemente do ambiente de Viçosa, esse caráter correlacionou-se negativamente com espessura de casca. Apesar de ser a mesma população avaliada em Viçosa e Miracema, o efeito ambiental pode ter resultado em diferenças quanto aos valores dos coeficientes de correlação.

Os valores dos coeficientes de correlação genéticoaditivas para a característica espessura de casca mostraram-se negativos com as variáveis peso de frutos, comprimento de frutos, largura de frutos e número de dias até a antese, indicando que a seleção de genótipos mais

Tabela 2 - Estimativas dos coeficientes de correlação fenotípica $\left(r_{F}\right)$ e dos coeficientes de correlação genético-aditivas $\left(\mathrm{r}_{\mathrm{GA}}\right)$, entre seis características de maracujá-amarelo para o ambiente de Miracema.

\begin{tabular}{clccccc}
\hline Características $^{1 /}$ & & $\mathrm{PF}$ & $\mathrm{CF}$ & $\mathrm{LF}$ & $\mathrm{EC}$ & $\mathrm{DA}$ \\
\hline $\mathrm{NF}$ & $\mathrm{r}_{\mathrm{F}}$ & $-0,28^{* *}$ & $-0,20^{* *}$ & $-0,07^{\mathrm{ns}}$ & $-0,03^{\mathrm{ns}}$ & $-0,06^{\mathrm{ns}}$ \\
& $\mathrm{r}_{\mathrm{GA}}$ & $-0,24^{* *}$ & $-0,15^{* *}$ & $0,22^{* *}$ & $0,12^{\mathrm{ns}}$ & $-0,07^{\mathrm{ns}}$ \\
$\mathrm{PF}$ & $\mathrm{r}_{\mathrm{F}}$ & - & $0,71^{* *}$ & $0,75^{* *}$ & $0,02^{\mathrm{ns}}$ & $0,13^{\mathrm{ns}}$ \\
& $\mathrm{r}_{\mathrm{GA}}$ & - & $0,71^{* *}$ & $0,80^{* *}$ & $-0,78^{* *}$ & $0,79^{* *}$ \\
$\mathrm{CF}$ & $\mathrm{r}_{\mathrm{F}}$ & & - & $0,47^{* *}$ & $-0,22^{* *}$ & $0,12^{\mathrm{ns}}$ \\
& $\mathrm{r}_{\mathrm{GA}}$ & & - & $0,36^{* *}$ & $-0,46^{* *}$ & $0,42^{* *}$ \\
$\mathrm{LF}$ & $\mathrm{r}_{\mathrm{F}}$ & & - & $0,10^{\mathrm{ns}}$ & $0,08^{\mathrm{ns}}$ \\
& $\mathrm{r}_{\mathrm{GA}}$ & & & $-0,50^{* *}$ & $0,59^{* *}$ \\
$\mathrm{EC}$ & $\mathrm{r}_{\mathrm{F}}$ & & & - & $-0,03^{\mathrm{ns}}$ \\
& $\mathrm{r}_{\mathrm{GA}}$ & & & & $-0,45^{* *}$ \\
\hline
\end{tabular}

${ }^{1 / N F}$ : número de frutos por planta; PF: peso de frutos, em gramas; CF: comprimento de frutos, em mm; LF: largura de frutos, em mm; EC: espessura de casca, em mm; DA: número de dias até a antese.

** Significativo a $1 \%$ de probabilidade pelo teste $\mathrm{t}$.

${ }^{\text {ns }}$ Não significativo. 
produtivos (maiores valores de peso, comprimento e largura de frutos) pode ocasionar uma minimização da espessura de casca.

De acordo com Cruz et al. (2004), considera-se aceitável proceder-se à análise conjunta dos ambientes quando o quociente entre o maior e o menor quadrado médio residual for menor do que 7. Sendo assim, os ambientes de Viçosa e Miracema foram avaliados conjuntamente para todas as características, pois apresentaram essa relação variando de 1,01 para largura de frutos (LF) a 2,41 para número de frutos por planta (NF), indicando o reduzido grau de heterogeneidade das variâncias residuais.

$\mathrm{Na}$ Tabela 3, encontram-se os valores dos coeficientes de correlação fenotípica e genético-aditivas para a análise conjunta dos ambientes de Viçosa e Miracema. Analisando-se esses valores, nota-se que não houve grandes divergências em relação à análise dos ambientes individualmente, o que era esperado devido à falta de interação entre genótipos e ambientes, encontrada na análise de variância conjunta entre esses ambientes.

A característica número de frutos por planta demonstrou estar associada negativamente com peso, comprimento e largura de frutos, e positivamente com espessura de casca, o que pode dificultar o trabalho de melhoramento, já que ganhos simultâneos em números de frutos e, peso, comprimento e largura de frutos são considerados de grande importância para uma população elite, assim, o uso de metodologias tais como os índices de seleção são recomendadas para a obtenção de ganhos seletivos.

Viana et al. (2003) estudaram as correlações simples e canônicas entre características de frutos de maracujáamarelo, avaliando cinco caracteres morfológicos relacionados à produção de frutos (número de frutos por planta, peso de frutos, comprimento de frutos, largura de frutos e espessura da casca) e três relacionados à qualidade dos frutos (grau brix, acidez e porcentagem de suco), verificando a existência de correlações genéticas positivas significativas, em ambos os ambientes, entre peso e largura de frutos, largura e comprimento de frutos, acidez e largura de frutos, e negativa entre grau brix e comprimento de frutos.

Comparando-se a característica peso de frutos com as demais, nota-se que ela apresentou correlações fenotípicas e genético-aditivas positivas com todas as demais, excluindo-se número de frutos por planta. Assim, o aumento nas dimensões do fruto pode resultar em diminuição da produção em números de frutos, de modo que métodos mais elaborados de seleção no melhoramento podem ser necessários para que se atinja uma população com alta produtividade e boas características comerciais. Ratifica-se, pois, a necessidade do uso de índices de seleção para obtenção de ganhos simultâneos em

Tabela 3 - Estimativas dos coeficientes de correlação fenotípica $\left(r_{F}\right)$ e dos coeficientes de correlação genético-aditivas $\left(\mathrm{r}_{\mathrm{GA}}\right)$, entre seis características de maracujá-amarelo para a análise conjunta dos ambientes de Viçosa e Miracema.

\begin{tabular}{clccccc}
\hline Características $^{1 /}$ & & $\mathrm{PF}$ & $\mathrm{CF}$ & $\mathrm{LF}$ & $\mathrm{EC}$ & $\mathrm{DA}$ \\
\hline $\mathrm{NF}$ & $\mathrm{r}_{\mathrm{F}}$ & $-0,34^{* *}$ & $-0,26^{* *}$ & $-0,21^{* *}$ & $-0,01^{\mathrm{ns}}$ & $-0,44^{* *}$ \\
& $\mathrm{r}_{\mathrm{GA}}$ & $-0,62^{* *}$ & $-0,55^{* *}$ & $-0,67^{* *}$ & $0,42^{* *}$ & $-0,09^{\mathrm{ns}}$ \\
$\mathrm{PF}$ & $\mathrm{r}_{\mathrm{F}}$ & - & $0,75^{* *}$ & $0,81^{* *}$ & $0,19^{* *}$ & $0,26^{* *}$ \\
& $\mathrm{r}_{\mathrm{GA}}$ & - & $0,88^{* *}$ & $0,85^{* *}$ & $0,17^{* *}$ & $0,55^{* *}$ \\
$\mathrm{CF}$ & $\mathrm{r}_{\mathrm{F}}$ & & - & $0,54^{* *}$ & $-0,15^{* *}$ & $0,33^{* *}$ \\
& $\mathrm{r}_{\mathrm{GA}}$ & & - & $0,67^{* *}$ & $0,05^{\mathrm{ns}}$ & $0,56^{* *}$ \\
$\mathrm{LF}$ & $\mathrm{r}_{\mathrm{F}}$ & & & - & $0,24^{* *}$ & $0,25^{* *}$ \\
& $\mathrm{r}_{\mathrm{GA}}$ & & & - & $0,15^{* *}$ & $0,66^{* *}$ \\
$\mathrm{EC}$ & $\mathrm{r}_{\mathrm{F}}$ & & & - & $-0,29 * *$ \\
& $\mathrm{r}_{\mathrm{GA}}$ & & & & $-0,34^{* *}$ \\
\hline
\end{tabular}

\footnotetext{
${ }^{1 /}$ NF: número de frutos por planta; PF: peso de frutos, em gramas; CF: comprimento de frutos, em mm; LF: largura de frutos, em mm; EC: espessura de casca, em mm; DA: número de dias até a antese.

** Significativo a $1 \%$ de probabilidade pelo teste $\mathrm{t}$.

${ }^{\text {ns }}$ Não significativo.
} 
características de importância econômica em maracujáamarelo.

\section{CONCLUSÕES}

A característica número de frutos por planta está associada negativamente com peso, comprimento e largura de frutos, e positivamente com espessura de casca.

A característica peso de frutos apresenta correlações fenotípicas e genético-aditivas positivas com todas as demais, excluindo-se número de frutos por planta.

Métodos de seleção mais apurados devem ser aplicados para a seleção dos genótipos, como os índices de seleção, já que algumas características importantes apresentam correlações negativas.

\section{REFERÊNCIAS BIBLIOGRÁFICAS}

COMSTOCK, R. E.; ROBINSON, H. F. The components of genetic variance in populations of biparental progenies and their use in estimating the average degree of dominance. Biometrics, North Carolina, v. 4, p. 254-266, 1948.

CRUZ, C. D.; CARNEIRO, P. C. S. Modelos biométricos aplicados ao melhoramento genético. Viçosa: UFV, 2003. v. $2,585 \mathrm{p}$.

CRUZ, C. D.; REGAZZI, A. J.; CARNEIRO, P. C. S. Modelos biométricos aplicados ao melhoramento genético. 3. ed. Viçosa: UFV, 2004. v. 1, 480 p.

FALCONER, D. S. Introdução à genética quantitativa. Viçosa: UFV, 1987. 279 p.

FURTADO, M. R. Alternativas de seleção no delineamento I de Comstock e Robinson, em milho. 1996. 94 f. Tese
(Doutorado em Genética e Melhoramento) - Universidade Federal de Viçosa, Viçosa, 1996.

GONÇALVES, G. M. Estimativas de parâmetros genéticos em características produtivas de maracujazeiro amarelo (Passiflora edulis f. flavicarpa), baseado no delineamento I. 2005. 91 f. Dissertação (Mestrado em Produção Vegetal) - Universidade Estadual do Norte Fluminense Darcy Ribeiro, Campos dos Goytacazes, 2005.

GONÇALVES, G. M.; VIANA, A. P.; BEZERRA NETO, F. V.; PEREIRA, M. G.; PEREIRA, T. N. S. Seleção e herdabilidade na predição de ganhos genéticos em maracujá-amarelo. Pesquisa Agropecuária Brasileira, Brasília, v. 42, n. 2, p. 193-198, 2007.

HALLAUER, A. R.; MIRANDA FILHO, J. B. Quantitative genetics in maize breeding. 2. ed. Ames: Iowa State University, 1988. $468 \mathrm{p}$.

ITI TROPICALS. Disponível em: <http:// Www.passionfruitjuice.com>. Acesso em: 20 mar. 2007.

PEREIRA, M. G.; AMARAL JÚNIOR, A. T. do. Estimation of genetic components in popcorn based on the nested design. Crop Breeding and Applied Biotechnology, Londrina, v. 1, n. 1, p. 3-10, 2001.

VIANA, A. P.; PEREIRA, T. N. S.; PEREIRA, M. G.; SOUZA, M. M. de; MALDONADO, J. F. M.; AMARAL JÚNIOR, A. T. do. Simple and canonic correlation between agronomical and fruit quality traits in yellow passion fruit (Passiflora edulis f. flavicarpa) populations. Crop Breeding and Applied Biotechnology, Londrina, v. 3, n. 2, p. 133-140, 2003. 